\section{Covid-19: Arthritis drugs improve survival in intensive care patients, shows study}

\author{
Jacqui Wise
}

Two rheumatoid arthritis drugs will now be used to treat the patients with severe covid-19 in intensive care, after new findings have shown that they significantly improve survival and cut the time spent in hospital by a week to 10 days.

Results from the REMAP-CAP trial showed that the interleukin 6 receptor antagonists tocilizumab and sarilumab reduced mortality from $35.8 \%$ to $27.3 \%$ compared with standard care-an absolute reduction of $8.5 \%$ and a relative reduction in mortality of $24 \% .{ }^{1}$ The benefit was seen when the drugs were given within 24 hours of patients entering intensive care and were in addition to a corticosteroid, such as dexamethasone.

Anthony Gordon, professor of anaesthesia and critical care at Imperial College London and chief investigator of the study, told a Science Media Centre briefing: "If we treat 12 patients it would save one life. This is a very big effect and really exciting."

The Department of Health and Social Care said that supplies of tocilizumab are already available in hospitals and that updated guidance would be issued to trusts across the UK encouraging its use. Either of the immune modulators could be used in addition to dexamethasone, which is already standard of care for patients in hospital with covid-19 receiving supplemental oxygen.

Promising interim results from the trial, involving 300 patients, were reported in November. ${ }^{2}$ The latest analysis of just over 800 patients is published as a preprint on medRxiv and has not yet been peer reviewed. The study included 353 patients assigned to tocilizumab, 48 to sarilumab, and 402 to standard care. Over $90 \%$ of patients were also treated with corticosteroids. The drugs are given as an hour long infusion. Most patients received a single infusion, with around 30\% needing a second dose.

The results were based on a combined outcome measure of survival and the length of time patients needed organ support. Compared with standard care, the odds ratios were 1.64 (95\% confidence interval 1.25 to 2.14 ) for tocilizumab and 1.76 (1.17 to 2.91) for sarilumab. Hospital mortality was $28 \%$ for tocilizumab and $22.2 \%$ for sarilumab, compared with $35.8 \%$ for the control group.

Steroids such as dexamethasone act as general anti-inflammatory agents, whereas tocilizumab and sarilumab block one specific receptor on the inflammatory pathway-interleukin 6.

The cost of treatment with either of the drugs is $€ 750-£ 1000$ (€830-€1105; \$1020-\$1360) per patient, substantially more than 10 days of treatment with the steroid dexamethasone, which would cost around
$£ 50$. But Gordon pointed out that a day in intensive care costs between £180o and £200o. "These drugs save lives and reduce the time spent in [intensive care] so are cost effective," he told the briefing.

Previous studies on tocilizumab have produced mixed results, although they have included patients that were less severely ill. Gordon said: "Previous trials using IL-6 receptor antagonists have showed no clear benefit on either disease progression or survival in covid-19 patients, but those studies included less severely ill patients and started treatment at different stages in the disease course." He added: “A crucial difference may be that in our study, critically ill patients were enrolled within 24 hours of starting organ support. This highlights a potential early window for treatment where the sickest patients may gain the most benefit from immune modulation treatment."

The RECOVERY trial, which is also looking at tocilizumab, includes patients who are less sick and has not yet published results. Its chief investigator Martin Landray, from the University of Oxford, said that the REMAP-CAP results were good news. "There are, of course, still unanswered questions. For example, exactly how well does tocilizumab work in different types of patients? And if given earlier, might it reduce the need for patients to require mechanical ventilation in the first place?” He said the RECOVERY trial should answer some of those questions.

Peter Horby, professor of emerging infectious diseases at the University of Oxford, said that the results give doctors more tools to fight covid-19. "Importantly, the findings of a benefit are in addition to corticosteroids, which were taken by $93 \%$ of the participants. This means we now have two drugs, which combined have a greater effect," he said.

Deputy chief medical officer Jonathan Van-Tam said: "This is a significant step forward for increasing survival of patients in intensive care with COVID-19. The data shows that tocilizumab, and likely sarilumab, speed up and improve the odds of recovery in intensive care, which is crucial for helping to relieve pressure on intensive care and hospitals and saving lives.”

The REMAP-CAP study is led by Imperial College London and the Intensive Care National Audit and Research Centre in the UK and University Medical Center in Utrecht. It is funded by the National Institute for Health Research in the UK and by the EU funded PREPARE consortium.

The REMAP-CAP trial is also evaluating the use of convalescent plasma for covid-19. A small randomised trial published in the New England Journal of Medicine, indicates that early treatment with 
convalescent plasma reduces the risk of progression to severe covid- 19 by $48 \% .^{3}$

1 REMAP-CAP Investigators, Gordon AC. Interleukin-6 receptor antagonists in critically ill patients with covid-19-preliminary report. MedRxiv [preprint]. https://www.medrxiv.org/content/10.1101/2021.01.07.21249390v1

2 Wise J. Covid-19: Critically ill patients treated with arthritis drug tocilizumab show improved outcomes, researchers report. BM/2020;371:m4530. . doi: 10.1136/bmj.m4530 pmid: 33214134

3 Libster R, Pérez Marc G, Wappner D, etalFundación INFANT-COVID-19 Group. Early high-titre plasma therapy to prevent severe ccovid-19 in older adults. N Engl / Med 2021;

doi: 10.1056/NEJMoa2033700. pmid: 33406353

This article is made freely available for use in accordance with BMI's website terms and conditions for the duration of the covid-19 pandemic or until otherwise determined by BMJ. You may use, download and print the article for any lawful, non-commercial purpose (including text and data mining) provided that all copyright notices and trade marks are retained. 